\title{
Desigualdad en hogares con adultos mayores en México, 2015
}

\author{
Inequality in homes with older adults in Mexico, 2015
}

\author{
Sergio Cuauhtémoc Gaxiola-Robles Linares, Daniel Lozano-Keymolen \\ y Bernardino Jaciel Montoya-Arce
}

\author{
Centro de Investigación y Estudios Avanzados de la Población \\ de la Universidad Autónoma del Estado de México
}

\section{Resumen}

El objetivo de este artículo es analizar la desigualdad entre los hogares con adultos mayores en México en el año 2015. La fuente de información es la Encuesta Intercensal 2015 del Instituto Nacional de Estadística y Geografía (INEGI). La propuesta metodológica de esta investigación se basa en la aplicación de un Análisis de Correspondencia Múltiple que permite estudiar la relación entre las variables de los hogares con adultos mayores en México con base en tres dimensiones: individual, hogar y contexto. Los resultados indican la presencia de desigualdades permanentes entre los hogares con personas con 60 años o más, además de diferencias importantes en los bienes, la estructura familiar y las ayudas gubernamentales, lo que impacta en las condiciones de vida de los adultos mayores de México en 2015.

Palabras clave: Hogares con adultos mayores, desigualdad, México, Análisis de Correspondencia Múltiple.

\section{Abstract}

The objective of the article is to analyze the inequality between households with older adults in Mexico in 2015. The source of information is the Intercensal Survey 2015 of the National Institute of Statistics and Geography (INEGI). The methodological proposal of this research is based on the application of a Multiple Correspondence Analysis that allows studying the relationship between the variables of households with older adults in Mexico based on three dimensions: individual, household and context. The results indicate the presence of permanent inequalities among households with people aged 60 and over, in addition to significant differences in assets, family structure and government aid, which impacts the living conditions of older adults in Mexico in 2015.

Keywords: Households with older adults, inequality, Mexico, Multiple Correspondence Analysis. 


\section{INTRODUCCIÓN}

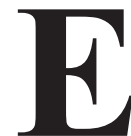

n los últimos años, la desigualdad y el envejecimiento demográfico son fenómenos que han impactado en los procesos sociales a través de cambios en las estructuras familiares y poblacionales. ${ }^{1}$ Lo anterior ha intensificado una serie de problemáticas en la sociedad como la pobreza, la polarización, el rezago social, entre otros.

En particular, en México la desigualdad se ha presentado históricamente y se caracteriza por ser altamente heterogénea, el Programa de las Naciones Unidas para el Desarrollo (PNUD) es un organismo internacional que expone la presencia de este fenómeno en México a través del Índice de Desarrollo Humano (IDH) en el que muestra diferencias relevantes a nivel entidad federativa (PNUD, 2011).

Por otro lado, según Esquivel (2015) en México la concentración de la riqueza es desigual, debido a que solo uno por ciento de la población posee 43 por ciento de toda la riqueza. En este informe se señala que México es el segundo país más desigual de los países de la Organización para la Cooperación y el Desarrollo Económico (OCDE). Una de las principales problemáticas que genera la desigualdad al interior de una sociedad es la falta de cohesión y convivencia entre sus integrantes.

En lo que respecta al envejecimiento demográfico, este fenómeno tiene sus antecedentes en el proceso de transición demográfica que se ha vivido en México, fenómeno caracterizado por la reducción de las tasas de mortalidad y natalidad de la población y cuyos efectos se expresaron en la disminución del crecimiento poblacional y en el incremento de las personas sobrevivientes a edades avanzadas.

En 1970 en México, la tasa de crecimiento poblacional era de 3.1 por ciento, para 1990 este indicador se reduce a dos por ciento y en la primera década del siglo XXI tanto la fecundidad como la mortalidad presentan niveles bajos, lo que se refleja en una tasa de crecimiento poblacional de 1.4 por ciento anual, esto implica una reducción importante para el crecimiento de la población mexicana y genera un incremento porcentual de la población envejecida.

Lo anterior impactó en un cambio en la distribución de los grupos de edad, por ejemplo, en 1990 la población de Adultos Mayores (AM) en México representaba 6.1 por ciento del total (INEGI, 1990). Para el año 2015 , los adultos mayores eran casi diez por ciento del total de la pobla-

1 Para esta investigación el envejecimiento demográfico se denota como el aumento en términos absolutos y porcentuales de la población de 60 años o más respecto al total de la población. 
ción, en números absolutos en el año 2015 había 12.4 millones de personas envejecidas (INEGI, 2015). Es decir, en 25 años el porcentaje de personas envejecidas casi se duplicó. Además, el incremento de los adultos mayores se va agudizar en los próximos años, por lo que resulta interesante conocer las problemáticas a las que se enfrentarán y los retos que tendrá que resolver el gobierno y la sociedad

En particular, este incremento se refleja también en el número de hogares con adultos mayores que en el año 2015 alcanzaban aproximadamente nueve millones de hogares y representaban 28.2 por ciento de los hogares en México, es decir, en uno de cada cuatro hogares en México habitaban al menos un adulto mayor, esto confirma una presencia importante de hogares con personas adultos mayores (INEGI, 2015).

En la actualidad, la presencia de la desigualdad y el incremento de las personas envejecidas generan una serie de demandas sociales que se concentran en aspectos como pensiones, protección social, atención a la salud, entre otras. La importancia de estos elementos para los adultos mayores radica en que permite mejorar las condiciones de vida de esta población, sin embargo, en México en el año 2014 solo uno de cada cinco adultos mayores contaba con acceso a pensión y protección social (Nava, Ham y Ramírez, 2016), lo que impacta en desigualdades relevantes en los hogares, debido a que al prevalecer la inseguridad económica (pensión) en las personas envejecidas, éstas se convierten en una carga familiar y ocasionan una dependencia al interior de los hogares que provoca fenómenos como la fragmentación familiar que se puede traducir en violencia y aislamiento de los adultos mayores (Melchiorre et al., 2013).

En este contexto, el presente artículo tiene como objetivo analizar la desigualdad entre los hogares con adultos mayores en México en el año 2015, mediante el uso de la información de la Encuesta Intercensal 2015 con la aplicación de un modelo de Correspondencia Múltiple para conocer las relaciones de desigualdad que se presentan entre los hogares con adultos mayores en México en dicho año.

El artículo consta de cinco apartados. En primer lugar, se presenta una revisión conceptual de la desigualdad, en segundo lugar, se exponen las principales características del envejecimiento demográfico. En tercer lugar, la metodología que consiste en la aplicación de un análisis por correspondencia múltiple. En cuarto lugar, el contexto sociodemográfico de los jefes de hogares con adultos mayores en México en 2015. Por último, se realiza el análisis de la desigualdad y los hogares con adultos mayores. 


\section{BREVE REVISIÓN CONCEPTUAL DE LA DESIGUALDAD}

La desigualdad ha sido un fenómeno constante a lo largo de la historia de la humanidad, consecuentemente su estudio ha permanecido vigente a través del tiempo. En el siglo XVIII, Rousseau (1999) analizó este fenómeno señalando la existencia de dos tipos de desigualdades: las desigualdades sociales o sustantivas que se construyen en función de las clases dominantes, y las desigualdades naturales o irrelevantes, que se relacionan con aspectos biológicos como el sexo y la edad. Sin embargo, la dicotomía planteada por el autor se ha hecho acreedora de diversas críticas. Por ejemplo, Beteille (1983) señala que la existencia de vínculos entre estas desigualdades mediante ciertas habilidades naturales ${ }^{2}$ generan diferencias sociales, por lo que se dificulta la posibilidad de establecer una clara separación entre ambas.

En los años recientes, la discusión sobre el concepto de desigualdad se ha desarrollado a partir de dos enfoques. Entre 1960 y 1970 distintos investigadores centraron su análisis en la desigualdad por clases, grupos o categorías sociales, uno de los más representativos fue Charles Tilly (2002). Sin embargo, en las postrimerías del siglo pasado cobró mayor fuerza una perspectiva alternativa que, en contraposición con la anterior, se concentraba en las desigualdades entre individuos. Así, una importante proporción de los investigadores se dedicaron a analizar las diferencias individuales (Davis y Moore, 1974; Sen, 1995), argumentando que el incremento de la heterogeneidad en la sociedad contribuyó a desvanecer la agrupación de la población en clases o categorías claramente identificables. Esta última vertiente continúa predominando en los estudios sobre la desigualdad realizados hoy en día.

Un ejemplo de lo anterior fue la teoría funcionalista que planteó la desigualdad como un fenómeno que ha existido históricamente en las sociedades y que dependía exclusivamente de los atributos que posee cada individuo. Este enfoque señalaba el carácter "funcional" de la desigualdad al contribuir a la reproducción de la sociedad promoviendo dos elementos fundamentales: la motivación y la recompensa. Kingsley y Wilbert, (1974), señalaban que ambos elementos legitimaban las desigualdades internas de la sociedad a través del sistema institucionalizado de recompensas diferenciadas que facilitaba la construcción del orden social. En particular, este tipo de desigualdades impactan directamente en los adultos mayores, según Alfageme (2000), las desigualdades individuales tienen un

\footnotetext{
2 A esto habría que incorporar un gran arsenal de literatura de los estudios de género donde se cuestiona las asignaciones de roles a las mujeres supuestamente debidas a "causas naturales".
} 
efecto en el ritmo y el modo de envejecer que se reflejan en discapacidades, dependencia, inseguridad económica y limitada atención médica.

En este sentido, Davis y Moore (1974: 96-97) mencionan que "la principal necesidad funcional que explica la presencia universal de la estratificación, es precisamente, la exigencia sentida por toda la sociedad de colocar y motivar a los individuos en la estructura social". Es importante señalar que estas recompensas pueden variar en su forma, aunque en su naturaleza continuarán siendo desigualdades sociales institucionalizadas.

Sin embargo, la teoría funcionalista de la desigualdad ha propiciado fuertes cuestionamientos. Una de las críticas más recurrentes a esta corriente ha sido su tendencia a estudiar la desigualdad al margen de las relaciones de poder que lleva implícitas. Ya desde inicio de los setenta Tumin (1974: 116) argumentaba que la presencia histórica de la desigualdad en las diferentes sociedades no es, necesariamente, un elemento indispensable para garantizar su funcionalidad. En palabras del autor, "la omnipresencia y la antigüedad de dicha desigualdad ha dado lugar a la suposición de que tal arreglo social debe ser inevitable y funcionalmente positivo".

Por otro lado, algunos estudios centrados en las diferencias por individuo (que no se circunscriben en la teoría funcionalista) han intentado caracterizar las igualdades - desigualdades que se presentan entre las personas. Por ejemplo, Turner (1986: 34) plantea cuatro tipos de igualdades:

1. Igualdad ontológica. Este tipo de igualdades se vinculan con aspectos morales y religiosos.

2. Igualdad de oportunidades. Se refiere al acceso igualitario (que no existe) a diversas instituciones sociales (educación, trabajo, salud), además estas igualdades se asocian a las recompensas y motivaciones en la estructura social.

3. Igualdad de condiciones. En este caso, las igualdades se identifican con aspectos que permitan o generen idénticas condiciones de vida en toda la población.

4. Igualdad de resultados. Esta noción se vincula con que independientemente de las condiciones o resultados, la población debe tener igualdad.

Es importante señalar que estas cuatro desigualdades se acentúan durante la vejez, debido a los diversos desarrollos personales y trayectorias laborales que generan grupos de adultos mayores con fuertes diferencias sociales, económicas, culturales y de salud que impactan en los niveles de vida de los AM. 
Complementando este análisis, durante los últimos años, Sen (1995) ha aportado elementos esenciales al estudio de las desigualdades individuales. A partir de la interrogante ¿desigualdad de qué?, el autor reconoce la existencia de una diversidad de características entre los individuos y plantea la importancia que éstas tienen dentro de la reproducción de la desigualdad. Así, Sen concluye que un elemento de considerable importancia dentro de las desigualdades individuales es la libertad, en palabras del autor "la libertad se encuentra entre los posibles campos de aplicación de la igualdad y la igualdad entre los posibles patrones de distribución de libertad" (Sen, 1995: 35).

Como se mencionó en un inicio, en contraposición a las teorías que analizan la desigualdad social enfocándose en las diferencias individuales, se encuentran aquellas investigaciones que estudian la desigualdad centrándose en la existencia de grupos, clases o categorías sociales que han condicionado y perpetuado las desigualdades a lo largo de la historia. A este grupo de autores pertenece Tilly (2000: 21) que plantea "las grandes y significativas desigualdades en las ventajas de que gozan los seres humanos corresponden principalmente a diferencias categoriales... más que a diferencias individuales en atributos, inclinaciones o desempeños".

Desde esta óptica, las diferencias categoriales constituyen desigualdades sistemáticas y duraderas, desigualdades que permanecen durante toda una vida y se reproducen generacionalmente. Estas desigualdades persistentes, se generan a través de dos mecanismos: la explotación y el acaparamiento de oportunidades. Según Tilly (2000: 23), el primero de estos mecanismos "actúa cuando personas poderosas y relacionadas disponen de recursos de los que extraen utilidades significativamente incrementadas mediante la coordinación del esfuerzo de personas ajenas a las que excluyen de todo el valor agregado por ese esfuerzo", mientras el segundo mecanismo "actúa cuando los miembros de una red categorialmente circunscripta ganan acceso a un recurso que es valioso, renovable, está sujeto a monopolio, respalda las actividades de la red y se fortalece con el modus operandi de ésta".

Aunado a lo anterior, esta vertiente muestra que las instituciones se conforman como un elemento central dentro de la reproducción e intensificación de las desigualdades, en la medida en que logran establecer un determinado orden social que perpetúa las diferencias existentes entre los diversos grupos o clases sociales. Tilly (2000: 21) menciona que "las desigualdades persistentes entre las categorías surgen porque las personas que controlan el acceso a recursos productores de valor resuelven problemas 
organizacionales acuciantes por medio de distinciones categoriales". En este contexto, una región que se caracteriza por tener sociedades con grandes desigualdades es América Latina que durante las décadas de 1960 y 1970 se desarrolló un profundo debate sobre la estratificación de la sociedad sustentado en los postulados de la teoría marxista. Una de las principales contribuciones fue realizada por Pinto (1998: 105 - 106), quien descifró las problemáticas que caracterizaban la estructura productiva de la región, al señalar que "a la luz de los cambios acarreados por la industrialización puede descomponerse la estructura productiva de la América Latina en tres grandes estratos. Por un lado, el "primitivo" cuyos niveles de productividad e ingreso por habitante probablemente son semejantes (y a veces inferiores) a los que primaban en la economía colonial y, en ciertos casos, en la precolombina. En el otro extremo, un "polo moderno", compuesto por las actividades de exportación, industriales y de servicios que funcionan a niveles de productividad semejante a los promedios de las economías desarrolladas, y, finalmente el "intermedio", que, de cierta manera, corresponde más cercanamente a la productividad media del sistema nacional".

De esta manera, el debate conceptual de la desigualdad social / laboral se plantea en dos corrientes de pensamiento. Por un lado, los autores que plantean a la desigualdad desde la perspectiva individual mediante aspectos como la recompensa. En contraste con estos autores, se plantea la desigualdad desde la perspectiva de grupos que se reproduce a través de la acumulación de privilegios.

En este sentido, la presente investigación optó por estudiar la desigualdad desde la perspectiva de grupos, debido a que posee tres grandes atributos. En primer lugar, hace posible redimensionar la importancia de los factores estructurales ${ }^{3}$ de la desigualdad, elementos que en los enfoques individualistas suelen quedar al margen. En segundo lugar, evidencia el carácter histórico de las desigualdades. En tercer lugar, permite analizar el impacto que tienen los grupos sobre los individuos. Por estos tres motivos, el presente trabajo ha optado por retomar dicho enfoque, en tanto permite analizar en forma más amplia el fenómeno del envejecimiento a través de la existencia de categorías en los hogares con adultos mayores fuertemente diferenciados entre sí.

${ }^{3}$ Los factores estructurales se definen como aquellos elementos que producen desigualdades sociales. Estos factores, determinan la conformación de clases sociales antagónicas, y es a través de estas clases que se expresan y reproducen las desigualdades sociales (y por lo tanto las laborales). A grandes rasgos, las clases sociales se definen de acuerdo con la relación de propiedad de un grupo respecto a los medios de producción, la función de éste dentro de la estructura productiva de una sociedad determinada y las relaciones sociales de producción que se entablan dentro de ella (Poulantzas, 1997). 
En esta lógica, los hogares se plantean como unidades domésticas que conservan desigualdades trascendentales que repercuten al interior de la sociedad. En particular, estas desigualdades impactan en las diferentes actividades que realiza la población envejecida, lo que a su vez produce y reproduce desigualdades intergeneracionales que se reflejan en la sociedad.

\section{ENVEJECIMIENTO DEMOGRÁFICO}

En términos demográficos, el siglo XXI se caracteriza por el constante crecimiento porcentual de la población de 60 años o más. Este aumento poblacional se le conoce como envejecimiento demográfico y se presenta en la mayoría de las sociedades latinoamericanas, lo anterior se debe fundamentalmente a la confluencia de dos factores: el descenso de la mortalidad y de la fecundidad.

El envejecimiento de la población conlleva una serie de transformaciones al interior de la sociedad debido a que este grupo tiene necesidades específicas en aspectos económicos, sociales, culturales y de salud que impactan en la dinámica de la sociedad y familiar.

Ante esta situación, el envejecimiento demográfico es un fenómeno que ha sido fuertemente estudiado desde hace muchos años, en particular se ha analizado desde un enfoque individual centrado en aspectos como la seguridad social, las pensiones, la calidad de vida, entre otras cosas. Sin embargo, en esta investigación se estudia el envejecimiento desde una perspectiva de hogares y familias con la finalidad de conocer con mayor profundidad las características y las diferencias que existen entre los hogares donde residen adultos mayores.

En este marco, los hogares con personas envejecidas (al menos un adulto mayor) son relevantes para el estudio de la población mayor a 60 años, debido a que el hogar y la familia es un espacio en el que se fortalecen/ debilitan los apoyos y las redes familiares de tal forma que permita mejorar/empeorar los niveles o la calidad de vida de los adultos mayores (Fu1ler-Iglesias y Antonucci, 2016), así resulta importante conocer la situación y la diferencia de los hogares envejecidos en México.

En las últimas décadas, se han presentado una serie de cambios en las estructuras, el tamaño y las dinámicas de los hogares, lo que ha agudizado las diferencias entre los adultos mayores e impacta en la presencia de la desigualdad en este grupo poblacional que en los próximos años tendrá un aumento sustancial de personas con una heterogeneidad de situaciones que acentúa la vulnerabilidad social de los adultos mayores. Con base en lo 
anterior, se plantea estudiar la relación entre diferentes tipos de hogares y variables en tres dimensiones: individual, familiar y contexto.

La primera dimensión hace referencia a las características individuales que permiten describir aspectos esenciales como la edad y el sexo del jefe de hogar. La segunda dimensión se refiere a los hogares y se asocia con bienes, tenencia y tipo de familia, estos aspectos permiten conocer el tipo de interacciones que se generan a nivel de los hogares. Por último, la tercera dimensión de las personas envejecidas son las características contextuales que proporcionan elementos del entorno del individuo y del hogar para establecer redes de apoyo social o familiar con base en la clase de vivienda y los apoyos gubernamentales. De esta manera, la relación entre los tipos de hogar y las dimensiones analizadas permite estudiar las diferencias que se presentan entre los cuatro grupos de hogares clasificados.

\section{Desigualdad y hogares}

En lo que corresponde a la relación entre desigualdad y hogares, diversos estudios (Cortes, 2013; Arriagada, 2002; Ariza y Oliveira, 2008) plantean vínculos entre el fenómeno de la desigualdad y los hogares. Por ejemplo, Arriagada (2002) estudia la desigualdad en los hogares a través del ingreso y el mercado laboral. En particular, analiza tres dimensiones: ingreso, tamaño y estructura del hogar.

En este orden de ideas, Ariza y Oliveira (2008) investigan la desigualdad social en seis países latinoamericanos que presentan transformaciones demográficas y socioeconómicas. En particular, estas autoras identifican que los cambios en los arreglos familiares impactan en las estructuras de los hogares y se vinculan con una serie de disimilitudes que permanecen al interior de las familias. En lo que respecta a los elementos socioeconómicos, las desigualdades se asocian con una serie de transformaciones económicas que se presentaron en América Latina y éstas acentuaron las desigualdades históricas de la región.

Para Cortés (2013), las desigualdades de los hogares en México durante el periodo de 1977 a 2000 se acentúan e impactan en una mayor concentración de la riqueza en solo un pequeño grupo de población, lo que genera la pérdida del poder adquisitivo en los hogares con mayores carencias.

Además, Jiménez y Ruedi (1998) plantean que en América Latina los hogares con personas envejecidas tienen condiciones socioeconómicas desiguales. Estas diferencias se vinculan con las trayectorias laborales de los adultos mayores, debido a que el trabajo genera condiciones materiales disímiles que se transfieren a los hogares y las familias 
De esta manera, estas investigaciones concluyen que la desigualdad está presente entre los hogares. En particular, en México el vínculo entre desigualdad y hogares se ha asociado con los ingresos familiares (Aguilar, 2000; Boltvinik y Hernández, 1999; Cortés, Hernández, Székely Vera, 2003), por lo que esta investigación propone incorporar el elemento del envejecimiento a la relación desigualdad y hogares.

\section{Metodología}

En este apartado se presentan los aspectos metodológicos que se utilizaron en esta investigación y que constan del desarrollo empírico, la unidad de análisis y la fuente de información. En lo que se refiere al desarrollo empírico se utilizaron dos instrumentos: una clasificación de los hogares y un Análisis de Correspondencia Múltiple (ACM).

\section{Desarrollo empírico}

Para el análisis empírico de este estudio se consideraron dos etapas. En la primera etapa se construyó una tipología de hogares basada en la condición de actividad de los adultos mayores de México en el año 2015. Esta clasificación consta de cuatro categorías asociadas con los quehaceres de las personas envejecidas: trabaja, no trabaja, jubilado o mixto.

En la segunda fase, se utiliza un Análisis de Correspondencia Múltiple (ACM) con variables categóricas asociadas con los bienes del hogar, el tipo de familia, la tenencia de la vivienda, la ayuda gubernamental, el tipo de hogar y el tipo de vivienda. Una de las ventajas del ACM es que permite mostrar a través de una gráfica la relación que existe entre estas variables y los cuatro tipos de hogares en México en el año 2015. La elección del $\mathrm{ACM}$ se debe a que permite cumplir con el objetivo del artículo que busca analizar las diferencias que existen entre los hogares con adultos mayores basados en tres dimensiones: individuales, familiar y contexto.

\section{Unidad de análisis}

Para esta investigación, se plantea analizar el envejecimiento demográfico desde una perspectiva de hogares, por lo que la unidad de análisis son los hogares de México en 2015. En particular, la investigación se concentra en los hogares con adultos mayores, es decir, aquellos hogares que tienen entre sus residentes al menos un adulto mayor. 


\section{Fuente de información}

En lo que respecta a la fuente de información, en esta investigación se utiliza la Encuesta Intercensal (EI) 2015 realizada por el Instituto Nacional de Estadística y Geografía (INEGI) que tiene una muestra de 6.1 millones de hogares y aproximadamente 22 millones de personas en México. El objetivo general de la EI es generar información estadística actualizada que proporcione estimaciones con calidad sobre el volumen, la composición y la distribución de la población y de las viviendas del territorio nacional, cuya desagregación geográfica sea por municipios, entidad federativa, localidades de 50 mil o más habitantes y a nivel nacional (INEGI, 2015). La elección de esta encuesta radica en dos aspectos. Por un lado, es la encuesta con mayor robustez estadística que existe en México por el tamaño de su muestra. Por el otro, la EI incluye las variables para realizar el estudio planteado. Es importante señalar que para esta investigación se utilizaron los datos expandidos de la Encuesta Intercensal con un resultado de 31.9 millones de hogares en México en el año 2015.

Una vez que se expone el análisis empírico, en la Figura 1 se muestra el planteamiento metodológico que consiste en presentar los elementos que se utilizaron para realizar y cumplir con el objetivo de este artículo. En primer término, se expone la unidad de análisis que corresponde a los hogares envejecidos, después se plantea la clasificación de hogares con adultos mayores, así como la aplicación del Análisis de Correspondencia Múltiple que permitió obtener los perfiles de los hogares con adultos mayores en México.

En la próxima sección se presenta el contexto general de los hogares en México en el año 2015 como preámbulo para el análisis estadístico, este contexto consiste en conocer el número de hogares totales en México y algunas características de los jefes y las jefas de hogar que expongan la situación sociodemográfica de los hogares con adultos mayores.

\section{CONTEXTO SOCIODEMOGRÁFICO DE LOS JEFES DE HOGARES CON ADULTOS MAYORES EN MÉXICO, 2015}

En esta sección se presenta una caracterización general de los hogares en México con la finalidad de mostrar algunas peculiaridades que coadyuven al análisis de las desigualdades entre los hogares con adultos mayores en México. En particular, se exponen las características sociodemográficas de los jefes y las jefas de hogar. 


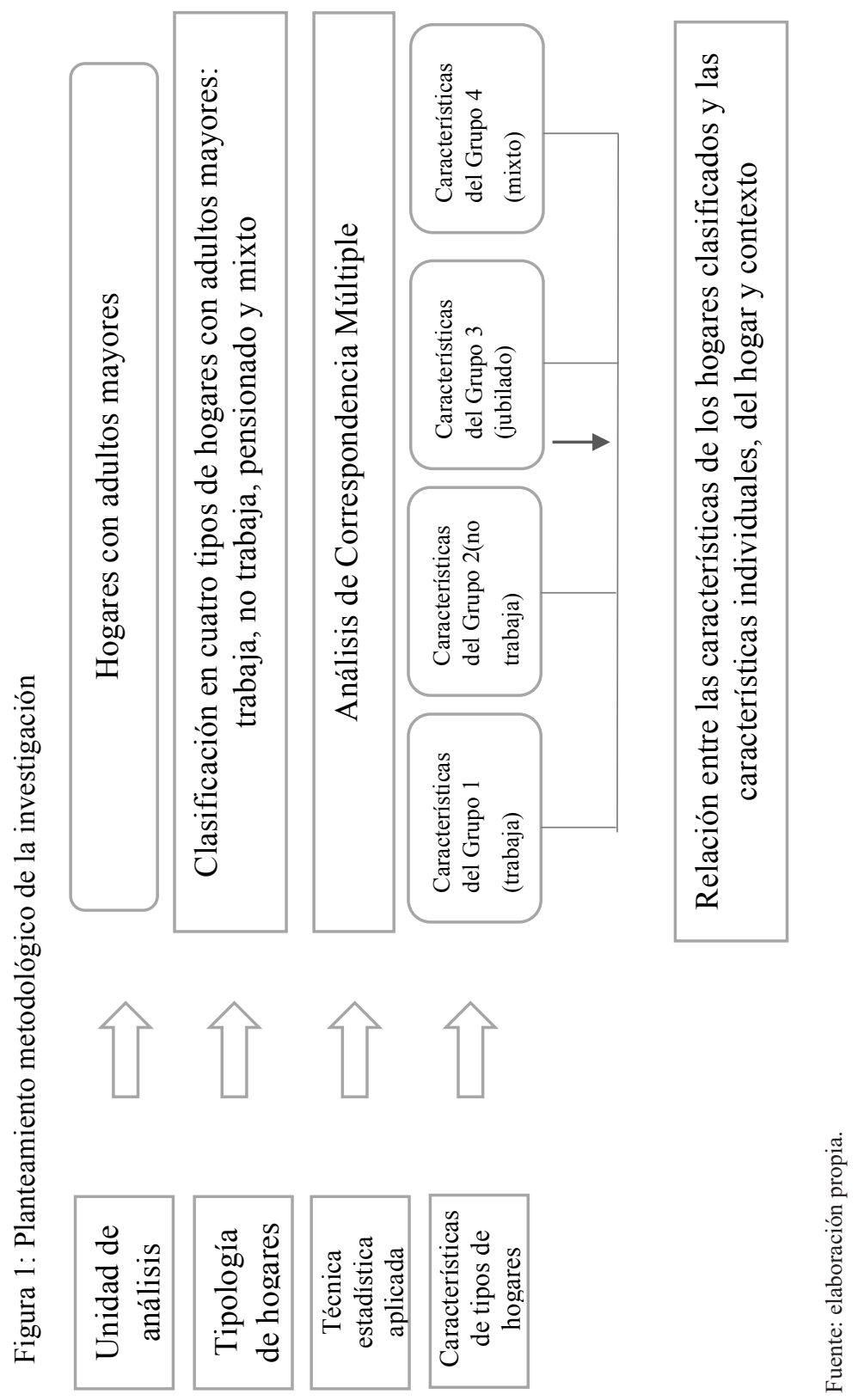


En el año 2015, el número de hogares que había en México era de casi 32 millones de los cuales nueve millones eran hogares con al menos un adulto mayor, éstos representaban aproximadamente 28 por ciento (véase Tabla 1). Lo anterior expone una fuerte presencia de hogares con adultos mayores que duplica el porcentaje de personas envejecidas a nivel individual (12 por ciento) en México y plantea la importancia de realizar un análisis del envejecimiento desde la perspectiva de los hogares para profundizar en el estudio de dimensiones asociadas con la estructura, la dinámica y las redes familiares.

En la Tabla 1 se expone la distribución porcentual de diversos aspectos sociodemográficos de los jefes y las jefas de hogar, la primera variable que se analiza es el sexo que muestra casi 40 por ciento de jefatura femenina en los hogares con adultos mayores, lo que es resultado de una mayor incorporación de las mujeres al mercado laboral y de una mayor sobrevivencia.

Por otro lado, en el año 2015 en México la variable de grupos de edad expone que 85 por ciento de los jefes de hogares con AM tienen edades mayores a 60 años, lo anterior otorga a los adultos mayores elementos de autoridad al interior del hogar e incrementa la participación de los adultos mayores en las decisiones relevantes del hogar (Tabla 1).

Por último, se presenta la variable del área geográfica en el que se expone que los hogares con adultos mayores se concentraban 76 por ciento en zonas urbanas, este porcentaje es similar para los hogares jóvenes (véase Tabla 1), por lo que la distribución de los hogares por área geográfica es similar en los dos tipos de hogares.

En términos generales, los hogares con adultos mayores exponen diversos aspectos relevantes que deben ser considerados para esta investigación como la alta presencia de jefatura femenina, que impacta de múltiples formas en la dinámica familiar. Por otro lado, la urbanización de México afecta de la misma manera a los hogares jóvenes y a los hogares con personas envejecidas, por lo que esta variable (que podría ser importante en el nivel de vida de los adultos mayores) expone que las personas envejecidas están fuertemente arraigadas a las zonas urbanas, contrario a lo que diversos estudios muestran con un mayor vínculo de los adultos mayores en zonas rurales marcados por aspectos culturales, tradicionales, posesión de propiedades e inclusive por cierta nostalgia del pasado (Monreal, Valle y Serna, 2009). 
Tabla 1: Distribución porcentual de características sociodemográficas en hogares, México, 2015

\begin{tabular}{lrrr}
\hline Características sociodemográficas & \multicolumn{2}{c}{ Hogares } \\
& Jóvenes & Envejecidos & Total \\
\hline Sexo & 74.4 & 62.3 & 71.0 \\
Hombre & 25.6 & 37.7 & 29.0 \\
Mujer & $22^{\prime} 889,901$ & $9{ }^{\prime} 059,808$ & $31^{\prime} 949,709$ \\
Total (100\%) & & & \\
Grupos de edad & 15.9 & 0.8 & 11.6 \\
12 a 29 & 84.0 & 13.7 & 64.1 \\
30 a 59 & 0.0 & 85.5 & 24.2 \\
60 o más & 0.1 & 0.0 & 0.1 \\
No especificado & $22^{\prime} 889,901$ & $99^{\prime} 059,808$ & $311^{\prime} 949,709$ \\
Total (100\%) & & & \\
Área geográfica & 21.0 & 23.8 & 21.8 \\
Rural & 79.0 & 76.2 & 78.2 \\
Urbano & $22^{\prime} 889,901$ & $9{ }^{\prime} 059,808$ & $31^{\prime} 949,709$ \\
\hline Total (100 \%)
\end{tabular}

Fuente: cálculos propios con base en la Encuesta Intercensal 2015 (INEGI, 2015).

\section{DESIGUALDAD Y HOGARES CON ADULTOS MAYORES EN MÉXICO, 2015}

Una vez que se expuso un contexto general de los hogares en México en el año 2015, en esta sección se muestra cómo se construye con mayor detalle la tipología de hogares para después aplicar el Análisis de Correspondencia Múltiple que permite conocer la relación que existe entre las variables seleccionadas y los tipos de hogares que se elaboraron.

\section{Tipología de hogares}

Como se mencionó anteriormente la tipología de hogares que se construyó en esta investigación se basa en la condición de actividad del adulto mayor, es decir, los hogares en México que contaban al menos con un adulto mayor se clasificaron en cuatro tipos de hogares: jubilado, trabaja, no trabaja o mixto.

La elección de la variable de condición de actividad de la persona envejecida para agrupar a los hogares se asocia con la trayectoria laboral $\mathrm{y}$ aspectos trascendentales de los adultos mayores y su familia como lo 
son la asistencia médica, la seguridad económica, los apoyos sociales, entre otros. Estos beneficios o derechos tienen un impacto sustancial en las condiciones materiales de los adultos mayores y en la relación familiar al interior de los hogares.

Para la clasificación de los hogares en México según la condición de actividad se utilizó la Encuesta Intercensal 2015 que permitió obtener cuatro tipos de hogares que se asocian con las actividades laborales de los adultos mayores. En términos generales las clasificaciones de los hogares envejecidos se describen a continuación.

En la Tabla 2 se muestran los cuatro tipos de hogares. En el primer grupo se encuentran los adultos mayores que reciben pensión o son jubilados, en estos hogares las personas envejecidas tienen garantizado aspectos esenciales para su sobrevivencia como lo es la seguridad económica (pensión) y la atención médica. Sin embargo, en México la pensión se asocia a ingresos económicos muy bajos que no permiten otorgar una completa seguridad económica a los pensionados.

Tabla 2: Clasificación de los hogares con base en la condición de actividad del adulto mayor

\begin{tabular}{ll}
\hline Condición de actividad & \multicolumn{1}{c}{ Características } \\
\hline Jubilado o pensionado & $\begin{array}{l}\text { Hogares con adultos mayores que reciben pensión o } \\
\text { jubilación. }\end{array}$ \\
Trabaja & $\begin{array}{l}\text { Hogares con adultos mayores que continúan insertos en } \\
\text { el mercado laboral }\end{array}$ \\
No trabaja & $\begin{array}{l}\text { Hogares con adultos mayores que no están insertos en el } \\
\text { mercado laboral. }\end{array}$ \\
Mixto & $\begin{array}{l}\text { Hogares con al menos dos adultos mayores que realizan } \\
\text { dos actividades diferentes. Por ejemplo, un adulto ma- } \\
\text { yor trabaja y el otro no trabaja. }\end{array}$ \\
\hline
\end{tabular}

Fuente: elaboración propia con base en la Encuesta Intercensal 2015 (INEGI, 2015).

El siguiente grupo se asocia con los adultos mayores que trabajan, lo que genera un desgaste físico y mental que impacta en la salud de las personas, es importante señalar que Nava y Ham (2014) mencionan que gran parte de los adultos mayores que continúan trabajando en México lo hacen por necesidades económicas que los obliga a realizar labores secundarias con bajas remuneraciones, de éstos aproximadamente 75 por ciento trabajan en la informalidad (Paz, 2010). El tercer grupo corresponde a los hogares con adultos mayores que no trabajan, este conjunto de hogares presenta mayor vulnerabilidad, debido a que los AM tienen una alta dependencia 
(económica, social, familiar) que en muchos casos genera exclusión social y fragmentación familiar de esta población (Aguilar, Álvarez y Lorenzo, 2011).

El último grupo se refiere a los hogares mixtos que constan de por lo menos dos adultos mayores que realicen actividades diferenciadas, en este tipo de hogares se presentan una heterogeneidad de situaciones que disminuyen la vulnerabilidad de las personas envejecidas, debido a que en el hogar al menos un adulto mayor realiza actividades económicas o es jubilado.

Con la clasificación de los cuatro tipos de hogares se procede a realizar la segunda etapa de la metodología que consiste en el Análisis de Correspondencia Múltiple (ACM) que posibilita estudiar la relación entre variables categóricas y los tipos de hogar a través de una visualización gráfica.

El Análisis de Correspondencia Múltiple tiene como objetivo estudiar la relación entre variables categóricas que se presentan en una tabla de contingencia (Greenacre, 1993). A grandes rasgos, el ACM transforma una tabla con información numérica a una gráfica que permite observar la relación que existe entre las variables estudiadas.

El proceso del ACM se sustenta en cuatro herramientas: perfiles, distancia, inercia y dimensiones. Los perfiles se asocian con las tablas de contingencia a través de ponderar los pesos de cada categoría para mostrarlos en el plano acorde a esta ponderación. La distancia tiene que ver con la distancia Ji cuadrada y sirve para poder calcular la inercia que es la longitud que existe entre los puntos de cada perfil hacia el centroide. Por último, las dimensiones son esenciales para el ACM, debido a que se relaciona con los ejes que se presentan en la gráfica y con la capacidad explicativa de las variables en la investigación.

En este sentido, las etapas que se realizan en un ACM se presentan a continuación. En la primera etapa se muestran tablas de contingencia de dos variables, en la que se exponen relaciones entre éstas. En segundo lugar, se muestran los perfiles que contienen renglones y columnas y que corresponden a frecuencias relativas de las variables analizadas. Por último, se muestra la gráfica de la tabla de contingencia que permite exponer las asociaciones que se presentan entre las variables.

Según Greenacre (1983) para realizar el ACM se requieren algunos elementos que se presentan a continuación:

1. Los perfiles del ACM son una representación matricial de las diferentes categorías de las variables estudiadas y se presentan según la distribución de las categorías. 
2. La masa es el peso que se asocia a los perfiles y con la frecuencia marginal de las categorías. En caso de que las variables tengan diferencias importantes en el número de observaciones, la masa permite compensar estas diferencias.

3. La distancia Ji cuadrada se relaciona con la inercia del ACM y tiene en cuenta la significancia de la asociación entre renglones y columnas. Según Clausen (1998: 11) "Esto implica que las categorías con pocas observaciones contribuyen relativamente más a la distancia entre puntos que las categorías con más observaciones".

4. En lo que respecta a la inercia su interpretación es geométrica y permite medir la dispersión de los perfiles. En este sentido, la inercia explica la relación entre las categorías de las variables.

5. Por último, las dimensiones se determinan conforme al número de categorías que se encuentren en las variables. Sin embargo, se sugiere considerar dos dimensiones con la finalidad de una mayor claridad en el análisis gráfico. Estas dimensiones tienen una capacidad explicativa de la relación entre las variables.

Ahora bien, una vez que se explican los diversos elementos del ACM se desarrolla el procedimiento para realizar esta técnica estadística. Sea una matriz $Z$ con $\mathrm{m}$ observaciones y $n$ variables categóricas, por lo que la matriz $Z$ tiene una dimensión de nxm y se denota de la siguiente manera:

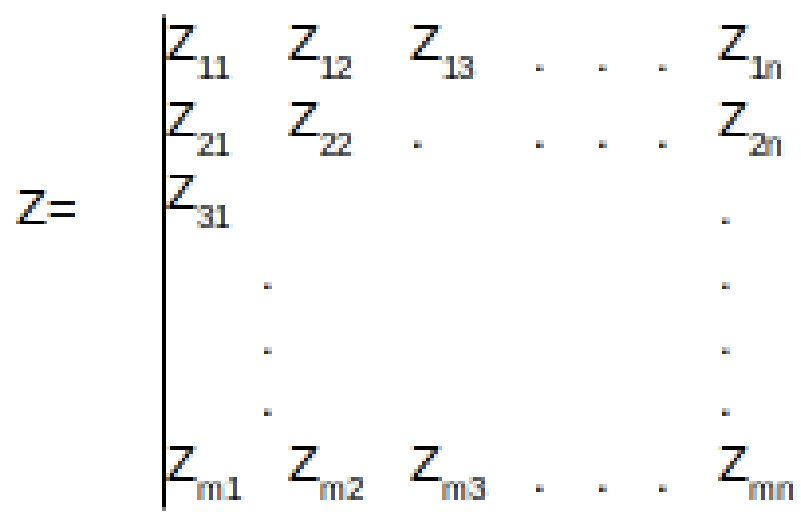

Una vez que se plantea $Z$ se debe obtener un matriz simétrica denominada $P$ a través de la recodificación en cero y uno de las categorías de las variables de $Z$, lo anterior con la finalidad de obtener la matriz de Burt que se define como $b=$ P' $P$ que se debe diagonalizar para obtener los vectores y valores propios a través de $\mathrm{S}=(1 / \mathrm{m}) * \mathrm{P}^{-1}$. Con $S$ es posible obtener los 
perfiles por renglones y columnas que generan las inercias y permiten graficar la relación entre las categorías de las variables en estudio, con base en lo anterior se exponen los principales resultados del ACM que se aplicó a las variables analizadas (Le Roux y Rouante, 2010).

Para esta investigación, las variables que se aplican en el ACM se estructuran en tres dimensiones que se asocian con aspectos centrales de los hogares y permiten conocer las relaciones que se forjan en los hogares con adultos mayores. La elección de las variables que se introducen en el ACM se debe a que posibilitan visibilizar diferencias entre los hogares analizados, por ejemplo, el tener una lavadora en un hogar genera ciertas diferencias con aquellos hogares que no poseen. En el caso de la variable tenencia de la vivienda, las familias que son dueños tienen certidumbre patrimonial sobre los hogares con vivienda rentada o prestada. En general, la elección de las variables se debe a que éstas contienen elementos que proporcionan diferencias relevantes que impactan en el nivel de vida de los adultos mayores. En la Tabla 3 se exponen las variables que se utilizan para el ACM.

Antes de aplicar el Análisis de Correspondencia Múltiple se presentan diversas tablas de contingencia de las variables analizadas y los hogares clasificados según la condición de actividad de los adultos mayores, con la finalidad de mostrar las relaciones entre los cuatro tipos de hogares y las variables seleccionadas (Tabla 3 ).

De esta manera, en la Tabla 4 se muestra que en el año 2015 había aproximadamente nueve millones de hogares envejecidos, de los cuales 13 por ciento eran hogares jubilados, esto es debido al bajo número de pensionados que existe en México (Nava, Ham y Ramírez, 2016), los hogares con adultos mayores que trabajaban eran 21 por ciento cifra similar a los hogares mixtos, por último, las viviendas en las que los adultos mayores no trabajaban eran aproximadamente 44 por ciento. Con esta distribución se puede observar una importante heterogeneidad en la clasificación de hogares que podría ser un indicio de hogares envejecidos con importantes diferencias. 
Desigualdad en hogares con adultos mayores en México, 2015/S.C. GAXIOLA ROBLES LINARES

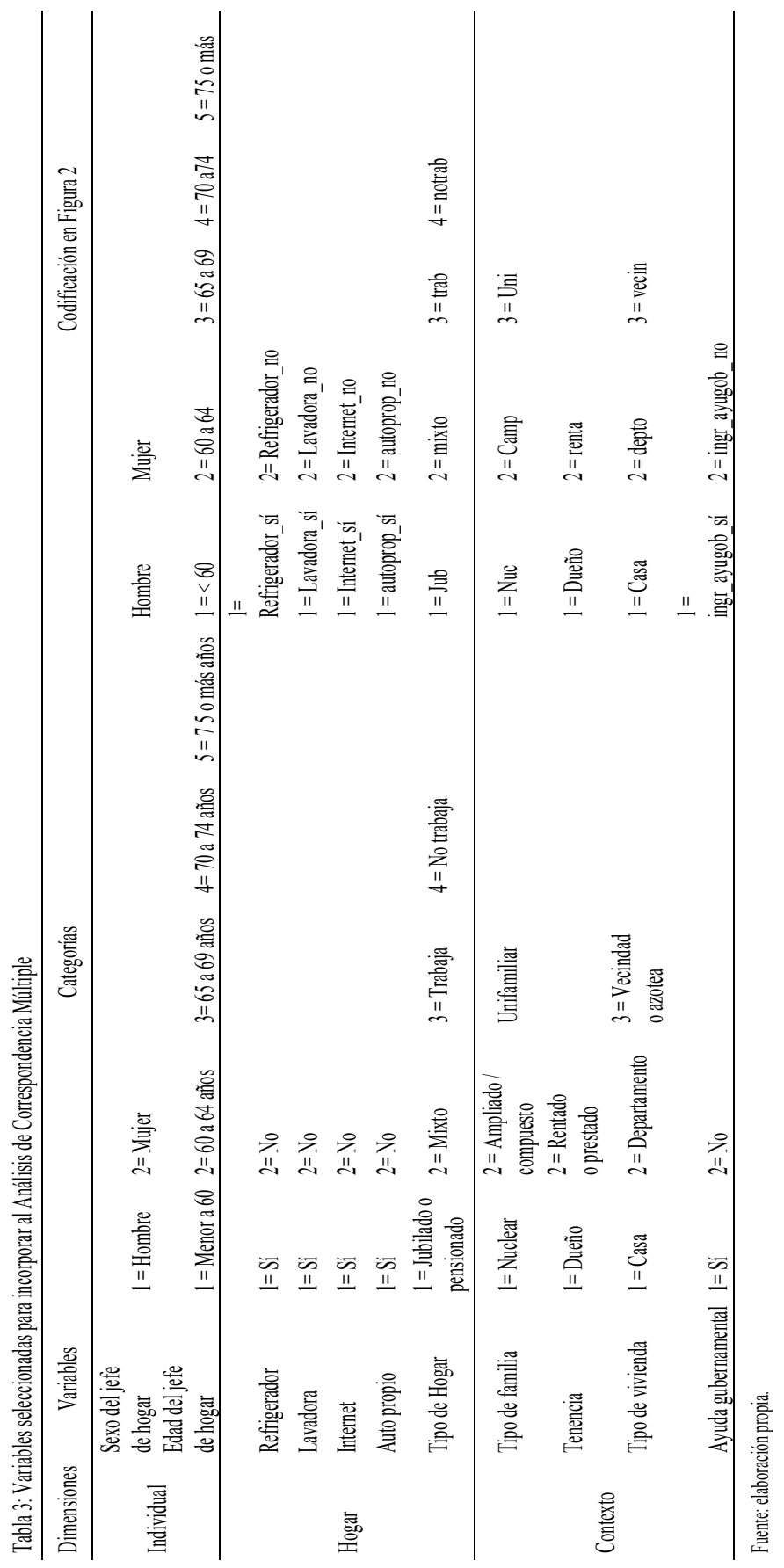


Tabla 4: Distribución porcentual por tipo de hogar con base en la condición de actividad, México, 2015

\begin{tabular}{lrrrrr}
\hline Distribución & \multicolumn{6}{c}{ Tipos de Hogares por condición de actividad } & \\
& Jubilado & Trabaja & No trabaja & Mixto & Total \\
\hline Absolutos & $1^{\prime} 210,776$ & 1'905,145 $^{\prime}$ & 3'960,068 $^{\prime}$ & 1'949,783 $^{\prime}$ & $9^{\prime} 025,772$ \\
Relativo & 13.4 & 21.1 & 43.9 & 21.6 & 100.0 \\
\hline
\end{tabular}

Fuente: cálculos propios con base en la Encuesta Intercensal 2015.

Tabla 5: Distribución porcentual de características sociodemográficas del jefe de hogar según por tipo de hogar, México, 2015

\begin{tabular}{lrrrr}
\hline $\begin{array}{l}\text { Características } \\
\text { sociodemográficas }\end{array}$ & \multicolumn{4}{c}{ Tipos de Hogares por condición de actividad } \\
& Trabaja & No trabaja & Jubilado & Mixto \\
\hline Sexo & & & & \\
Hombre & 67.7 & 33.3 & 54.0 & 48.4 \\
Mujer & 32.3 & 66.7 & 46.0 & 51.6 \\
Total (100\%) & 1 '210,776 & 1'905,145 & 3'960,068 & 1'949,783 \\
Grupos de edad & & & & \\
60 a 64 & 54.5 & 22.0 & 29.6 & 31.3 \\
65 a 69 & 24.7 & 20.8 & 23.5 & 27.8 \\
70 a 74 & 11.8 & 19.2 & 18.1 & 19.0 \\
75 o más & 9.1 & 38.0 & 28.8 & 21.9 \\
Total (100\%) & 1 '210,776 & 1'905,145 & 3'960,068 & 1 1'949,783 \\
\hline
\end{tabular}

Fuente: cálculos propios con base en la Encuesta Intercensal 2015.

\section{Características individuales}

Para las características individuales se eligieron las variables de sexo y grupos de edad de los jefes de hogar con la finalidad de conocer aspectos sociodemográficos de los jefes de hogar. En el año 2015 en México con referencia a la variable sexo se presentan disimilitudes entre los hogares trabaja y no trabaja, por ejemplo, en el hogar de adultos mayores que trabajan 67 por ciento son hombres jefes de hogar y 33 por ciento son mujeres jefas de hogar, en contraste los hogares que no trabajan solo alcanzan 32 por ciento y 68 por ciento como jefes y jefas de hogar respectivamente (véase Tabla 5). Para los hogares mixto y jubilados, la Tabla 5 muestra que la variable sexo del jefe de hogar tiene una presencia similar, en particular los hogares mixtos cuentan con un mayor porcentaje de mujeres como je- 
fas de hogar, en el caso de los hogares jubilados sorprende un porcentaje de 46 por ciento para las mujeres, debido a que la jubilación se vincula a los hombres porque tienen las posibilidades de beneficiarse con trayectorias laborales más extensas.

Lo anterior expone que en los hogares con adultos mayores se continúan reproduciendo patrones diferenciados de género que relaciona el hombre al trabajo y a la jubilación, actividad que mejora el nivel de vida a través de la seguridad económica.

En contraste, las mujeres tienen una predominancia en los hogares que no trabajan en donde se invisibilizan actividades como el trabajo doméstico y el de cuidados (Trujillo, Mroz, Piras, Ángeles y Tran, 2012), en este sentido se presentan desigualdades permanentes de género entre los hogares con adultos mayores.

La segunda variable que se expone son los grupos de edad de los jefes de hogar, en el que los hogares que trabajan tienen altos porcentajes de jefes de hogar en las edades de 60 a 64 y 65 a 69 años, en contraste los hogares que no trabajan son los más longevos, debido a que 38 por ciento de los jefes de hogar se concentran en las edades de 75 años y más (véase Tabla 5). Para los hogares mixtos y jubilados la distribución es más equitativa entre los grupos de edad de los jefes de hogar. En términos generales, en la variable de grupos de edad de los jefes de hogar los más afectados resultan ser los más longevos (no trabajan), debido a que el deterioro físico y la condición de no trabajar incrementa la dependencia de los adultos mayores lo que los convierte en personas más vulnerables en múltiples situaciones. En este marco, estas características individuales permiten mostrar desigualdades permanentes asociadas a los diferentes tipos de hogares, situación que agudiza las condiciones de vida y materiales durante la vejez e impactan en las dinámicas de esta población.

\section{Características del hogar}

La segunda dimensión que se analiza son las características del hogar, en este caso las variables estudiadas son los diversos bienes de la vivienda (lavadora, refrigerador, internet y auto propio), así como el tipo de familia. En la Tabla 6 se expone la distribución porcentual de las características del hogar con los cuatro tipos de hogares clasificados. La primera variable que se estudia es la tenencia de refrigerador según los resultados obtenidos el análisis se puede dividir en dos grupos de hogares: el primero se vincula a los hogares mixtos y jubilados que contaban con más de 90 por ciento 
con refrigerador, el segundo grupo que contiene a los hogares trabaja y no trabaja tenían 80 por ciento refrigerador.

Tabla 6: Distribución porcentual de los bienes,tipo y tipo familia, según tipo de hogar, México, 2015

\begin{tabular}{|c|c|c|c|c|}
\hline \multirow[t]{2}{*}{ Bienes } & \multicolumn{4}{|c|}{ Tipos de Hogares por condición de actividad } \\
\hline & Trabaja & No trabaja & Jubilado & Mixto \\
\hline \multicolumn{5}{|l|}{ Refrigerador } \\
\hline $\mathrm{Si}$ & 84.6 & 81.9 & 96.5 & 91.3 \\
\hline No & 15.2 & 17.8 & 3.3 & 8.4 \\
\hline No especificado & 0.2 & 0.3 & 0.2 & 0.2 \\
\hline Total $(100 \%)$ & $1 ’ 905,145$ & 3’960,068 & $1 ’ 210,776$ & $1^{\prime} 949,783$ \\
\hline \multicolumn{5}{|l|}{ Lavadora } \\
\hline $\mathrm{Si}$ & 67.6 & 62.2 & 85.3 & 79.0 \\
\hline No & 32.2 & 37.5 & 14.4 & 20.7 \\
\hline No especificado & 0.2 & 0.3 & 0.3 & 0.3 \\
\hline Total $(100 \%)$ & $1 ’ 905,145$ & 3’960,068 & $1 ’ 210,776$ & $1 ' 949,783$ \\
\hline \multicolumn{5}{|l|}{ Automóvil propio } \\
\hline $\mathrm{Si}$ & 41.9 & 29.4 & 55.3 & 50.3 \\
\hline No & 57.6 & 70.1 & 44.2 & 49.3 \\
\hline No especificado & 0.4 & 0.4 & 0.4 & 0.4 \\
\hline Total $(100 \%)$ & $1 ’ 905,145$ & 3'960,068 & $1 ’ 210,776$ & $1 ' 949,783$ \\
\hline \multicolumn{5}{|l|}{ Internet } \\
\hline $\mathrm{Si}$ & 31.3 & 20.1 & 48.3 & 34.3 \\
\hline No & 68.2 & 79.5 & 51.3 & 65.3 \\
\hline No especificado & 0.3 & 0.4 & 0.4 & 0.4 \\
\hline Total $(100 \%)$ & $1 ’ 905,145$ & 3’960,068 & $1 ’ 210,776$ & $1 ' 949,783$ \\
\hline \multicolumn{5}{|l|}{ Tipo de familia } \\
\hline Nuclear & 42.9 & 31.6 & 36.7 & 57.0 \\
\hline Ampliado /compuesto & 35.9 & 48.9 & 37.1 & 41.2 \\
\hline Unipersonal /corresidente & 19.9 & 18.2 & 25.0 & 0.0 \\
\hline No especificado & 1.3 & 1.3 & 1.1 & 1.7 \\
\hline Total $(100 \%)$ & $1 ’ 905,145$ & 3'960,068 & $1 ’ 210,776$ & 1'949,783 \\
\hline
\end{tabular}

Fuente: cálculos propios con base en la Encuesta Intercensal 2015. 
Por otro lado, en lo que corresponde a la posesión de lavadora en los cuatro grupos de hogares clasificados se observa una mayor heterogeneidad comparado con el refrigerador, debido a que el rango de tenencia de lavadora varía entre 62 por ciento en los hogares no trabajan y 85 por ciento de los hogares jubilados (véase Tabla 6). Es importante señalar que la lavadora es considerada un elemento vital para los hogares debido a que disminuye en gran medida el trabajo doméstico que en muchos casos son realizados por las mujeres adultas mayores y que genera una serie de desigualdades y enfermedades (Lawlor et al., 2002; Archer et al., 2013). Para esta variable se exponen diferencias considerables entre los hogares estudiados que impactan en la dinámica cotidiana de los adultos mayores por los múltiples efectos generados en los quehaceres del hogar.

La tercera variable que se analiza es el auto propio que es un elemento central para la movilidad de los adultos mayores, en este caso las diferencias entre los tipos de hogar son preocupante debido a que más de 50 por ciento de los hogares jubilados y mixtos contaban con automóvil propio, en contraste los hogares no trabajan solo 30 por ciento tenía vehículo (véase Tabla 6). En lo que respecta a los hogares trabaja cerca de 40 por ciento contaban con automóvil.

En esta variable se mantiene y acentúa la desigualdad de los hogares, debido a que solo uno de cada tres hogares que no trabajan tienen auto propio, lo que implica una limitación importante para la movilidad y el traslado de las personas envejecidas hacia lugares básicos como citas médicas.

Por otro lado, las comunicaciones familiares son vitales para mantener la unidad y la cohesión familiar y en la actualidad una de las herramientas tecnológicas más utilizadas es el servicio de Internet, en la Tabla 6 se expone que los cuatro tipo de hogares tienen porcentajes menores a 50 por ciento, el hogar que no trabaja tiene los porcentajes más bajo con 20 por ciento, en contraste los hogares jubilados casi 50 por ciento si cuentan con el servicio, para los hogares mixtos y jubilados solo 30 por ciento cuentan con Internet.

El bajo porcentaje (menor a 50 por ciento) con servicio de internet en los cuatro tipos de hogares se asocia con dos aspectos relevantes: por un lado, el retraso tecnológico generacional que restringe su uso, por el otro, el acceso a estos servicios resulta costoso para gran parte de la población. Lo anterior limita en gran medida la utilización de estos recursos para mantener una comunicación constante entre los familiares de los adultos mayores y en dado caso que los utilicen en muchos casos las personas envejecidas se vuelven dependientes tecnológicos, debido a que el uso del 
Internet y de las computadoras para los adultos mayores depende del apoyo por parte de familiares más jóvenes.

En lo que respecta a la variable de composición familiar incluye las categorías de hogares nucleares, ampliadas, compuestas, unipersonales y corresidentes ${ }^{4}$ para los hogares mixtos 95 por ciento se concentran en familias ampliadas y nucleares, en lo que respecta los hogares jubilados, trabaja y no trabaja solo 70 por ciento se concentraban en dicha categoría (véase Tabla 6). A grandes rasgos se puede mencionar que tres de cada cuatro adultos mayores en México viven con sus familiares, es importante esta convivencia debido a que una de las causas de morbilidad y mortalidad en los adultos mayores es la depresión y el aislamiento social (Choi et al., 2015).

Según la Tabla 6, aproximadamente 20 por ciento de los hogares jubilados, trabaja y no trabaja se concentraban en los hogares unipersonales, estos adultos mayores resultan ser altamente vulnerables, debido a la falta de interacción familiar y social, además estos hogares se asocian con el aislamiento y la falta de apoyo hacia la población adulta mayor.

A grandes rasgos, las variables que corresponden al hogar exponen la presencia de diferencias relevantes entre los hogares envejecidos, en particular esta dimensión plantea dos grupos de hogares, por un lado, el grupo de hogares jubilados y mixtos que son los que cuentan con las mejores condiciones, el otro grupo de hogares conformado por los que trabajan y no trabajan muestran condiciones materiales desfavorables.

\section{Características del contexto}

Por último, se analizan variables asociadas con las características del contexto, en la Tabla 7 se presenta la distribución de los hogares envejecidos por tenencia y tipo de la vivienda, así como ayuda gubernamental.

En la variable de tenencia de la vivienda, casi 80 por ciento de los hogares envejecidos en México tienen viviendas propias, este porcentaje es similar en los cuatro tipos de hogares, lo que podría vincularse con una cuestión generacional y se asocia con que los adultos mayores tuvieron mayores posibilidades de adquirir bienes inmuebles (Tabla 7). Es importante señalar que a nivel nacional el porcentaje de personas que son due-

\footnotetext{
${ }^{4}$ Según el INEGI, un hogar nuclear está conformado por el jefe(a) y cónyuge; jefe(a) e hijos(as); jefe(a), cónyuge e hijos(as), un hogar ampliado está conformada por un hogar nuclear y al menos otro pariente, o por un jefe(a) y al menos otro pariente, un hogar unipersonal está formado por una persona y un hogar corresidente está formado por dos o más personas sin parentesco con el jefe del hogar.
} 
ñas de la vivienda que habitan es aproximadamente 67 por ciento (INEGI, 2015).

Situación similar sucede con la variable clase de vivienda en el que 90 por ciento de los hogares con AM en México son casas, lo que implica beneficios en el nivel de vida de los adultos mayores, debido a que vivir en una casa habitación tiene ciertas ventajas como espacios para la convivencia, el desenvolvimiento y la movilidad de las personas envejecidas (véase Tabla 7).

Tabla 7: Distribución porcentual de la tenencia, tipo y ayuda gubernamental en las viviendas por tipo de hogar, México, 2015

\begin{tabular}{|c|c|c|c|c|}
\hline \multirow[t]{2}{*}{ Características hogar } & \multicolumn{4}{|c|}{ Tipos de Hogares por condición de actividad } \\
\hline & Trabaja & No trabaja & Jubilado & Mixto \\
\hline \multicolumn{5}{|l|}{ Tenencia vivienda } \\
\hline Propia & 77.9 & 81.3 & 82.0 & 87.7 \\
\hline Renta / prestada & 19.7 & 16.7 & 16.1 & 10.7 \\
\hline No Especificado & 0.6 & 0.7 & 0.6 & 0.7 \\
\hline Total $(100 \%)$ & 1'905,145 & $3^{\prime} 960,068$ & $1 ’ 210,776$ & 1'949,783 \\
\hline \multicolumn{5}{|l|}{ Tipo de vivienda } \\
\hline Casa & 92.3 & 95.6 & 89.8 & 94.6 \\
\hline Departamento & 4.8 & 2.4 & 8.2 & 3.9 \\
\hline Vecindad / azotea & 1.7 & 0.8 & 0.9 & 0.5 \\
\hline No Especificado & 1.2 & 1.2 & 1.0 & 1.0 \\
\hline Total $(100 \%)$ & $1 ’ 905,145$ & 3’960,068 & $1 ’ 210,776$ & $1 ' 949,783$ \\
\hline \multicolumn{5}{|l|}{ Ayuda gubernamental } \\
\hline Sí & 32.3 & 56.7 & 18.7 & 44.7 \\
\hline No & 67.2 & 42.7 & 80.5 & 54.6 \\
\hline No Especificado & 0.6 & 0.6 & 0.8 & 0.6 \\
\hline Total $(100 \%)$ & $1^{\prime} 905,145$ & $3^{\prime} 960,068$ & $1^{\prime} 210,776$ & 1'949,783 \\
\hline
\end{tabular}

Fuente: cálculos propios con base en la Encuesta Intercensal 2015.

Por último, se expuso la ayuda gubernamental, en la Tabla 7 se muestra a los hogares envejecidos con diferencias importantes entre los cuatro tipos de hogares analizados, por ejemplo, 56 por ciento de los hogares que no trabajan reciben ayudas del gobierno, por lo que resultan ser los hogares 
más beneficiados, en contraste los que menos reciben esta ayuda (20 por ciento) son los hogares jubilados. En lo que respecta a los hogares mixtos 44 por ciento reciben ayuda gubernamental, por último, los hogares que trabajan sólo uno de cada tres recibe este tipo de ayudas. Lo que se expone en esta variable es una fuerte heterogeneidad que se traduce en diferencias relevantes entre los cuatro tipos de hogares.

En resumen, las características contextuales, plantean aspectos relevantes en torno a los hogares con AM, por ejemplo, se observa que en el año 2015 en México gran parte de las personas envejecidas habitaban en casas propias lo que expone una homogeneidad entre estas variables, sin embargo, en lo que corresponde a la variable ayuda gubernamental se observan diferencias relevantes entre los hogares envejecidos.

Los análisis de estas tres dimensiones de los hogares envejecidos clasificados por condición de actividad del adulto mayor exponen aspectos relevantes y discordantes que se presentan en estos hogares. Por un lado, se observa diversos elementos heterogéneos como los bienes en el hogar, la estructura familiar, así como las ayudas gubernamentales que reciben. Por el otro, se exponen variables con resultados homogéneos como el tipo y la tenencia de la vivienda. De esta manera, los resultados de esta investigación identifican aspectos que son centrales para mantener la desigualdad entre los hogares envejecidos y que deberían ser atendidos para cohesionar a la población adulta mayor y mejorar las condiciones de vida.

\section{Aplicación del ACM}

Los resultados del ACM permiten mostrar los vínculos entre los cuatro tipos de hogares y las variables seleccionadas. Como se mencionó anteriormente, un elemento relevante para el ACM son las dimensiones que explican las relaciones que existen entre las variables estudiadas. En la Tabla 8 se muestra que las primeras dos dimensiones explican aproximadamente 70 por ciento de las relaciones entre variables, por lo que esta investigación retoma estas dimensiones para realizar la gráfica del ACM, según Algañaraz (2016) el porcentaje de explicación de la variabilidad debe concentrarse en las primeras dos dimensiones para tener la certidumbre de que la gráfica tenga la capacidad explicativa sobre las variables estudiadas.

Una de las ventajas que tiene el ACM es la gráfica que genera en la que se pueden observar las relaciones entre las variables elegidas, en particular en esta investigación se analiza la relación de las dimensiones individual, familiar y contexto con los cuatro tipos de hogares: jubilados, trabaja, no trabaja o mixto. 
Desigualdad en hogares con adultos mayores en México, 2015/S.C. GAXIOLA ROBLES LINARES

Tabla 8: Valor de las dimensiones del Análisis de Correspondencia Múltiple.

\begin{tabular}{ccc}
\hline Dimensión & Porcentaje & Acumulado \\
\hline 1 & 42.3 & 42.3 \\
2 & 28.5 & 70.8 \\
3 & 12.4 & 83.2 \\
4 & 8.6 & 91.8 \\
5 & 3.5 & 95.3 \\
6 & 2.1 & 97.4 \\
7 & 1.6 & 99 \\
8 & 0.5 & 99.5 \\
9 & 0.3 & 99.8 \\
10 & 0.2 & 100.0 \\
\hline
\end{tabular}

Fuente: cálculos propios con base en la Encuesta Intercensal, 2015.

En la Figura 2 se exponen las categorías de las variables elegidas, por ejemplo, la categoría que se muestra con el nombre de "jub" se refiere a que la condición de actividad del adulto mayor de esos hogares es "jubilado", una relación más detallada de estas categorías se puede encontrar en la Tabla 3. Es importante señalar que para el ACM la relación entre las categorías de cada variable se basa en la cercanía / lejanía. Es decir, entre mayor cercanía de las categorías en el plano existe una mayor relación. En contraste entre más distancia la relación se desvanece.

Los resultados muestran diferentes relaciones entre las variables que ayudan a conocer las características que tienen los cuatro tipos de hogares estudiados. Por ejemplo, el grupo 1 está conformado por aquellos hogares en el que los adultos mayores trabajan y tienen entre 60 y 69 años, el jefe de hogar es hombre, sí cuentan con los bienes del hogar, viven en una familia ampliada o compuesta, y la vivienda es propia (véase Figura 2).

En la Figura 2 se muestra el grupo 2 que corresponde a los hogares con adultos mayores que no trabajan estos hogares se caracterizan por tener jefas de hogar, rentan en la vivienda que residen, los AM son personas con más de 75 años, cuentan con pocos bienes y reciben ayuda del gobierno, estos hogares se ubican en zonas con menos de 50 mil habitantes.

En lo que respecta al grupo 3 son los hogares con adultos mayores pensionados o jubilados que se ubican en el en el primer cuadrante de la Figura 2 cerca de áreas con más de 50 mil habitantes, cuentan con la mayoría de los bienes en el hogar, no reciben ayudas gubernamentales. 
Figura 2: Análisis de Correspondencia Múltiple según tipos de hogar con adultos mayores en México, 2015

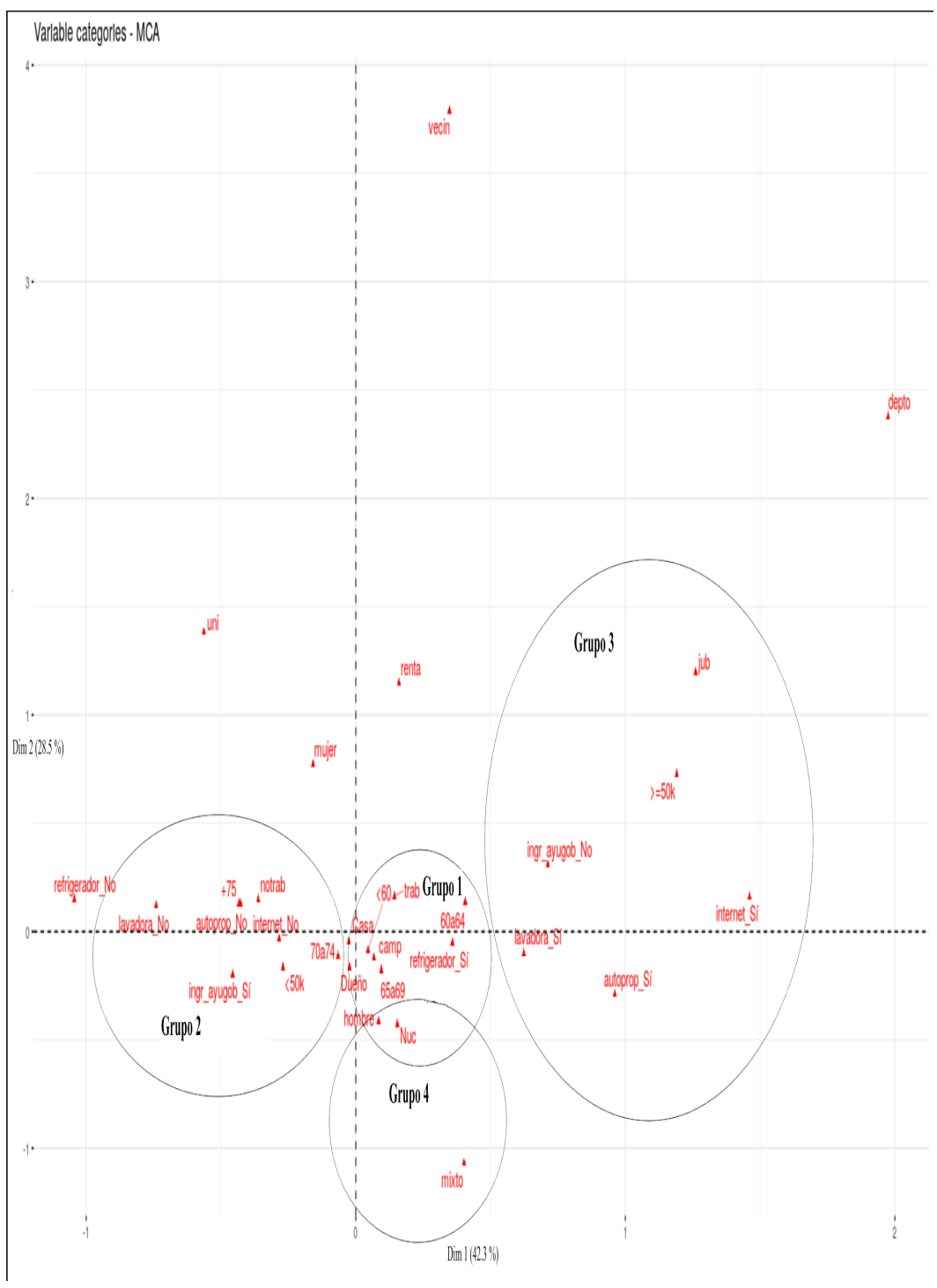

Fuente: elaboración propia con base en la Encuesta Intercensal 2015. 
En este caso, los hogares con adultos mayores jubilados son los que cuentan con mayores privilegios, ya que tienen mayor seguridad económica y derechohabiencia para la atención médica.

Por último, el grupo 4 que se conforma por los hogares mixtos que se asocian con hogares nucleares, cuentan con bienes y el jefe de hogar es hombres (véase Figura 2). Es importante señalar que los hogares jubilados y mixtos son los que se encuentran más alejados de la mayoría de las categorías, debido a que en la conformación del ACM son los que se encuentran con menos casos por lo que el peso que tienen en la gráfica hace que se alejen del centro donde se encuentran la gran parte de las categorías.

\section{Principales desigualdades en los hogares con adultos mayores en México en 2015}

En términos generales, los resultados mostrados en la Figura 2 exponen que los cuatro tipos de hogares tienen características diferenciadas, por ejemplo, se observa que los hogares más privilegiados cuentan con los bienes en el hogar, es decir, tienen en cierta medida seguridad económica (jubilados). En contraste, los hogares con adultos mayores que no trabajan tienen diversas carencias al interior del hogar que impactan en las condiciones materiales y sociales del adulto mayor. En lo que respecta a los hogares mixtos, la figura plantea limitadas relaciones con este grupo de hogar que tiene como sus principales características que son hogares nucleares. Por último, los hogares con AM que trabajan cuentan con aspectos relevantes como que son los de menor edad entre los AM (60 a 69 años) y residen en casas propias recursos que inciden en mejorar el nivel de vida de las personas envejecidas.

Aunado a lo anterior, los grupos de hogares analizados por dimensiones expresan una importante heterogeneidad en las tres dimensiones (individuales, hogar y contexto) que permite identificar dos conjuntos de hogares, por un lado, el grupo 2 (no trabajan) que se relacionan con mujeres longevas y presentan mayores carencias como la falta de algunos bienes en el hogar, así como ayudas gubernamentales. Por el otro lado, los grupos de hogar 1 y 3 que se asocian con ciertos privilegios como tener automóvil, bienes en el hogar y con ingresos económicos y sin ayuda gubernamental.

En resumen, los hogares mexicanos con adultos mayores en 2015 presentan desigualdades estructurales que se vinculan con la condición de actividad de las personas envejecidas en México, estas desigualdades se asocian con la trayectoria laboral de los adultos mayores e inciden en el nivel de vida y las condiciones materiales de esta población, lo que genera 
que los AM enfrenten la vejez con grandes diferencias. En particular estas desigualdades afectan a las mujeres por las dificultades para desarrollar una trayectoria laboral que les permita contar durante la vejez con elementos básicos como la seguridad económica de tal forma que mejoren sus niveles de vida.

\section{ConClusiones}

Los resultados que se obtuvieron en esta investigación permiten afirmar una serie de aspectos sobre los perfiles de los hogares con adultos mayores y la desigualdad que existe entre ellos en México en el año 2015. En particular se exponen dos elementos que se consideran los de mayor relevancia.

En primer lugar, las tres dimensiones analizadas exponen diferencias entre los hogares con personas envejecidas que se catalogan como desigualdades por grupos y que se vinculan a las desigualdades permanentes. En este sentido, los hogares que no trabajan son los que presentan mayores vulnerabilidades en las tres dimensiones, en contraste los hogares jubilados o pensionados que se asocian a los hogares mejor posicionados. Lo anterior impacta en el nivel de vida de los adultos mayores, por ejemplo, mientras gran parte de los hogares jubilados cuentan con bienes en el hogar, habitan en casas, son dueños de la vivienda, en contraste los hogares que no trabajan presentan menos elementos económicos para enfrentar los últimos años de sus vidas, lo que incrementa la vulnerabilidad de esta población.

En segundo lugar, las tres dimensiones analizadas permitieron mostrar una heterogeneidad vinculada con la desigualdad por grupos que expone situaciones complejas para los adultos mayores en México, debido a que los hogares con AM se agrupan en dos modalidades. Por un lado, aquellos que cuentan con la seguridad económica. Por el otro, aquellos hogares que cuentan con redes familiares fuertes.

\section{REFERENCIAS BIBLIOGRÁFICAS}

Aguilar, G., 2000, Desigualdad y pobreza en México. ¿Son inevitables? México: Universidad Nacional Autónoma de México, Instituto de Investigaciones Económicas y Sociales, Instituto Politécnico Nacional, Centro de Investigaciones Económicas, Administrativas y Sociales, Miguel Ángel Porrúa.

Aguilar, J., Álvarez, J., y Lorenzo, J., 2011, "Factores que determinan la calidad de vida de las personas mayores", in International Journal of Developmental and Educational Psychology, 4(1),161-168. 
Alfageme, A., 2000, “Algunas desigualdades en el envejecer de los ancianos españoles de los años noventa", en Revista Española de Investigaciones Sociológicas. 92, 93-112.

Algañaraz, V., 2016, "El Análisis de Correspondencias Múltiples como herramienta metodológica de síntesis teórica y empírica", en Revista Latinoamericana de Metodología de las Ciencias Sociales. 6(1), 2-17.

Archer, E., Shook, R., Thomas, D., Church, T., Katzmarzyk, P., Hébert, J., Mclver, K., Hand, G., Lavie, C., and Blair, S., 2013, “45-Year Trends in Women's Use of Time and Household Management Energy Expenditure", in PloS One, 8(2), e56620. doi:10.1371/journal.pone.0056620.

Ariza, M., y De Oliveira, O., 2008, "Familias, pobreza y desigualdad social en Latinoamérica: una mirada comparativa", en Revista Latinoamericana de Población, 1 (2): 73 - 98.

Arriagada, Irma, 2002, "Cambios y Desigualdad en las Familias Latinoamericanas”, en Revista de la CEPAL, ISSN 1682-0908, núm. 77, pp 143-161.

Béteille, A., 1983, The idea of natural inequality and other essays. Delhi: Oxford.

Boltvinik, J., y Hernández, E., 1999, Pobreza y distribución del ingreso en México. México: Siglo XXI.

Choi, H., Irwin, M. y Cho, H., 2015, "Impact of social isolation on behavioral health in elderly: systematic review", in World Journal of Psychiatry, 5(4), 432438. doi:10.5498/wjp.v5.i4.432.

Clausen, S., 1998, Applied correspondence analysis an introduction, Sage, London.

Cortés, F., 2013, "Medio siglo de desigualdad en el ingreso en México", en Economía UNAM, 10 (29), 12-34.

Cortés, F., Hernández, D., Hernández, E., Székey, M., y Vera, H., 2003, “Evolución y características de la pobreza en México en la última década del siglo XX", en Economía Mexicana. Nueva Época, XII (2): 295 - 325.

Davis, K., y Moore, W., 1974, Algunos fundamentos de la teoría de la estratificación. En La Desigualdad social: teorías de la estratificación y la movilidad sociales, compilado por Stern, Claudio, México: Secretaría de Educación Pública, $84-106$.

Esquivel, G., 2015, Desigualdad extrema en México. Concentración del poder económico y político. México: Oxfam.

Fuller-Iglesias, H., y Antonucci. T., 2016, "Convoy of social support in Mexico: examining socio-demographic variation", in International Journal of Behavioral Development, 40(4), 324-333.

Greenacre, M., 1993, Correspondence Analysis in Practice, Academic Press Limited, USA.

INEGI, 1990, Censo de Población y Vivienda 1990. Resultados principales. México.

INEGI, 2015, Encuesta Intercensal, 2015. Microdatos. México. 
Jiménez, L., y Ruedi, N., 1998, "Determinantes de la desigualdad entre los hogares urbanos", en Revista CEPAL, 66(1): 53 - 72.

Lawlor, D., Taylor, C., Bedford, R., and Ebrahim, S., 2002, "Is housework good for health? Levels of physical activity and factors associated with activity in elderly women. Results from the British Women's Heart and Health Study", in Journal of Epidemiology \& Community Health, 56(6), 473-478. doi:10.1136/jech.56.6.473.

Le Roux, B., Rouante, H., 2010, Multiple Correspondence Analysis. Series: Quantitative Applications in the Social Sciences. SAGE.

Melchiorre, M., Chiatti, C., Lamura, G., Torres, F., Stankunas, M., Lindert, J., Ioannidi-Kapolou, E., Barros, H., Macassa, G., y Soares, J., 2013, "Social support, socio-economics status, health and abuse among older people in seven European countries", in PloS ONE, 8(1): e54856.

Monreal, P., Valle, A. y Serda, B., 2009, "Los grandes olvidados: Las personas mayores en el entorno rural”, en Psychosocial Intervention, 18(3), 269-277.

Nava, I. y Ham, R., 2014, "Determinantes de la participación laboral de la población de 60 años o más en México”, en Papeles de Población, 20 (81), 59-87.

Nava, I., Ham, R. y Ramírez, B., 2016, “Seguridad económica y vejez en México”, en Revista Latinoamericana de Población. 10(19), 169 - 190.

Paz, J., 2010, Envejecimiento y empleo en América Latina y el Caribe, Ginebra: International Labour Office, Documento de Trabajo núm. 54.

Pinto, A., 1998, "Naturaleza e implicaciones de la "heterogeneidad estructural" de América Latina", en Cincuenta años del pensamiento en la CEPAL, Textos seleccionados, vol. 2. Chile: CEPAL / FCE, $547-567$.

PNUD, 2011, Informe sobre Desarrollo Humano México 2011. Equidad del gasto público: derechos sociales universales con subsidios focalizados.

Poulantzas, N., 1997, Poder politico y clases sociales en el Estado capitalista. México: Siglo XXI.

Rousseau, J.J., 1999, Discurso sobre el origen de la desigualdad entre los hombres. California: Prometeo.

Sen, A., 1995, Nuevo examen de la desigualdad. Madrid: Alianza.

Tilly, C., 2000, La desigualdad persistente. Buenos Aires: Manatial.

Trujillo, A., Mroz, T., Piras, C., Angeles, G., y Tran, N., 2012, "Caregiving and elderly health in Mexico", in International Journal of Health Services, 42(4), 667694.

Tumin, M.,1974, "Un análisis crítico del trabajo de Davis y Moore”, en La Desigualdad social: teorías de la estratificación y la movilidad sociales. México: Secretaría de Educación Pública.

Turner, B., 1986, Equality. England: Taylor \& Francis. 


\section{RESUMEN CURRICULAR DE LOS AUTORES}

\section{Sergio Cuauhtémoc Gaxiola Robles Linares}

Actuario por la Facultad de Ciencias de la Universidad Nacional Autónoma de México. Maestro en Estudios de Población por la Facultad Latinoamericana de Ciencias Sociales (sede México). Doctor en Estudios de Población por El Colegio de México. Profesor de Tiempo Completo del Centro de Investigación y Estudios Avanzados de la Población de la Universidad Autónoma del Estado de México (UAEM) y miembro del Sistema Nacional de Investigadores (SNI). Docente en programas de licenciatura de la UAEM. Ha publicado varios artículos. Líneas de investigación: envejecimiento, mercados de trabajo y pobreza.

Dirección electrónica: srobles99@gmail.com

\section{Daniel Lozano Keymolen}

Es doctor en Estudios de Población por El Colegio de México. Se desempeña como profesor e investigador de tiempo completo en el Centro de Investigación y Estudios Avanzados de la Población de la Universidad Autónoma del Estado de México. Ha publicado diversos artículos de investigación sobre el perfil de la salud y acerca de los procesos de desigualdad en el estado de salud de las personas en edades avanzadas. Pertenece al Sistema Nacional de investigadores Nivel I de Conacyt de México.

Dirección electrónica: dlozano@colmex.mx

\section{Bernardino Jaciel Montoya Arce}

Es Licenciado, Maestro y Doctor en Sociología por la Universidad Nacional Autónoma de México (UNAM). Pertenece al Sistema Nacional de Investigadores Nivel I, cuenta con Perfil Deseable PROMEP. Actualmente es Coordinador del Centro de Investigación y Estudios Avanzados de la Población de la Universidad Autónoma del Estado de México (CIEAP-UAEM). Entre sus publicaciones recientes se encuentran Demografía indígena en el Estado de México (coautor), 2013; Análisis demográfico del envejecimiento en el Estado de México (compilador), UAEM, 2011 y "La educación indígena en el Estado de México", en Papeles de Población, 2013.

Dirección electrónica:bjmontoyaa@uaemex.mx

Artículo recibido el 18 de diciembre de 2019 y aprobado el 18 de febrero de 2020. 